\title{
„Nur wenn wir richtig gut sind, werden wir unsere Position behaupten können.“
}

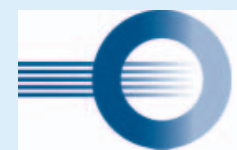

AG Herz- und Gefäßdiagnostik

in der Deutschen Röntgengesellschaft

Prof. Dr. David Maintz, Vorsitzender des Vorstands der AG Herz- und Gefäßdiagnostik der Deutschen Röntgengesellschaft, gibt im Interview einen Einblick in die aktuelle Herzbildgebung und markiert die aus seiner Sicht wichtigen Themen und Projekte für 2021.

Herr Professor Maintz, Sie haben im September $\mathbf{2 0 2 0}$ den Vorstandsvorsitz der AG Herz- und Gefäßdiagnostik übernommen und damit in einer berufspolitisch gerade für die Herzbildgebung vergleichsweise unruhigen Zeit. Wie schätzen Sie die allgemeine "Großwetterlage“ ein und welche Schlussfolgerungen ziehen Sie daraus?

Die allgemeine Großwetterlage wird natürlich gerade von COVID-19 bestimmt. In
Bezug auf die Herzbildgebung ist die Wetterlage sonnig, aber mit drohenden Gewittern: Nie war die Bedeutung und die Evidenz für Herz-CT und -MRT höher, aber das weckt auch Begehrlichkeiten.

Die vermehrt auftretenden Versuche, der Radiologie die Herzbildgebung in Teilen oder in Gänze streitig zu machen, kommen sicherlich nicht von ungefähr, so ist beispielsweise der Stellenwert der Herz$\mathrm{CT}$ in der Diagnose der KHK durch aktuelle Leitlinien erheblich gewachsen. Auch wird allgemein erwartet, dass die HerzCT und möglicherweise auch die HerzMRT in absehbarer Zeit Eingang finden werden in den Leistungskatalog der GKV. Inwieweit beeinflusst diese Entwicklung die Arbeit der AG?
Es wird allerhöchste Zeit, dass die Herz-CT und -MRT als Kassenleistung abrechenbar werden. Deswegen unterstützen wir die Bestrebungen des Berufsverbandes (BDR) in diese Richtung so gut wir können. Um die Verbreitung der Methoden im Alltag in Deutschland noch besser einschätzen und belegen zu können, haben wir eine Umfrage unter den Radiologinnen und Radiologen der Universitätsklinika, Krankenhäuser und unter Niedergelassenen durchgeführt - mit interessanten Ergebnissen: 147 Einrichtungen, die an der Umfrage teilgenommen haben, haben 2019 circa 77000 Untersuchungen erbracht. Wie werden die Zahlen erst aussehen, wenn die Leistungen eine angemessene Vergütung erfahren!? Insgesamt ist bei Radiologinnen und Radiologen in Deutschland viel Expertise in der Herzbildgebung vorhanden. Die AG Herz- und Gefäßdiagnostik ist mit 1538 Mitgliedern besonders stark, darunter finden sich 841 Mitglieder mit Q1-Status, 224 Mitglieder mit Q2-Status und 142 Mitglieder mit Q3-Status. 73 Zen- 


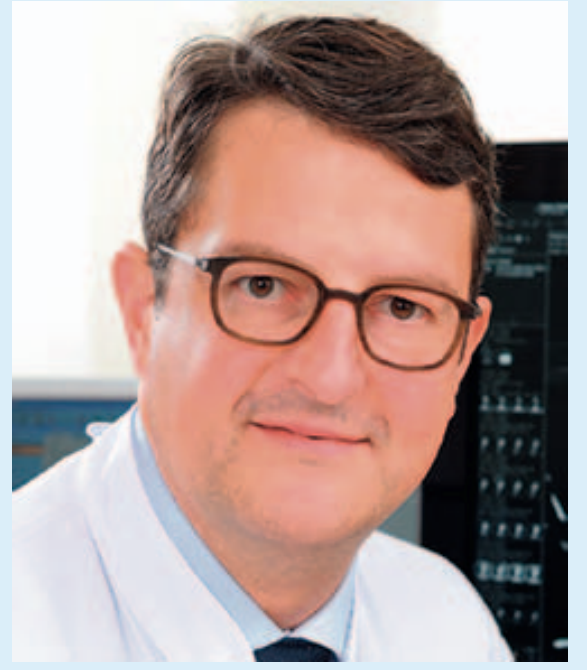

Prof. Dr. David Maintz

tren sind zudem als DRG-Zentren für kardiovaskuläre Bildgebung zertifiziert.

Das Jahr 2020 stand ganz im Zeichen der Corona-Pandemie. Inwieweit hatte diese Auswirkungen auf die Aktivitäten der AG Herz- und Gefäßdiagnostik - beispielsweise im Bereich Fortbildungsveranstaltungen oder Zertifizierungen?

Zunächst einmal mussten wir für unsere eigenen regelmäßigen Vorstandssitzungen auf ein digitales Format umstellen. Das hat sich als sehr konstruktiv erwiesen. Gleiches galt für die Fortbildungen. Q-Kurse wurden online organisiert, unter der Leitung von Frau PD Dr. Katharina Fischbach. Zuletzt fanden der Q1-Kurs CT am 23.11.2020 und der Q2-Kurs CT am 26.11.2020 unter der Leitung von PD Dr. Christopher Schlett und Prof. Dr. Christian Ritter statt - mit 198 beziehungsweise 113 Teilnehmerinnen und Teilnehmern! Das Feedback war überwältigend. Besonders geschätzt wird das LiveOnlineformat und es wurde wiederholt der Wunsch geäußert, dieses auch nach der Corona-Pandemie beizubehalten. Auch die Q-Prüfungen können jetzt remote angeboten werden. Dank der Arbeit von Dr. Sebastian Reinartz und Frau Melek Mirzanli stehen hierfür überarbeitete Prüfungsmodule bereit. Außerdem werden in der aktuellen Situation klinikinterne CME-Punkte anerkannt (zunächst befristet bis zum 30.06.2021) und die Regelungen für die Q1-3-Zertifizierungen wurden vereinheitlicht, im Detail zu finden auf der AG-Homepage.
Welche Themen und Projekte standen für die AG darüber hinaus in 2020 im Fokus?

Da fällt mir spontan die strukturierte Befundung ein. Wir haben in einer größeren interdisziplinären Runde mit Kardiologinnen und Kardiologen, Kinderkardiologinnen und -kardiologen sowie Herzchirurginnen und -chirurgen Templates für die wichtigsten Herzuntersuchungen im Konsens erarbeitet. Inzwischen liegen diese frei verfügbar zum Download auf der Homepage befundung.drg.de vor. Die wissenschaftlichen Hintergründe für die Befundvorlagen werden in Publikationen in Wissenschaftsjournalen veröffentlicht, diejenigen für Kardiomyopathien sind bereits erschienen, die anderen folgen bald.

\section{INFOKASTEN}

Die Publikation „Strukturierte Befundung in der Schnittbilddiagnostik des Herzens: Befundvorlagen für die MRT bei Kardiomyopathien “ können Sie online abrufen unter:

https://www.thieme-connect.com/pro ducts/ejournals/abstract/10.1055/a0998-4116

Das Motto für den Deutschen Röntgenkongress 2021 lautet „Intelligenz vernetzen“. Inwieweit lebt und transportiert die AG Herz- und Gefäßdiagnostik bereits heute diesen Vernetzungsgedanken zum Beispiel mit anderen Arbeitsgemeinschaften in der DRG oder auch interdisziplinär, das heißt mit anderen Fachgesellschaften?

Dafür gibt es einige sehr gute Beispiele: Die strukturierte Befundung habe ich erwähnt. Sie wird in enger Zusammenarbeit mit der AGIT, unter anderem mit PD Dr. Daniel Pinto dos Santos und Dr. Malte Sieren, betrieben und technisch umgesetzt. Hinzu kommen Fortbildung und Lehre: Die digitale Lernplattform der DRG, conrad, wurde und wird mit starker Beteiligung der AG Herzund Gefäßdiagnostik aufgebaut. Eine regelmäßige Interaktion findet auch statt mit der neu gegründeten AG Gesundheitspolitische Verantwortung unter dem Vorsitz von Dr. Lars Benjamin Fritz und mit dem BDR, um unsere gemeinsamen Interessen zu besprechen und zu vertreten. Aber auch mit den Kolleginnen und Kollegen der Kardiologie, Kinderkardiologie und Herzchirurgie gibt es einen regelmäßigen inhaltlichen Austausch, um immer wieder neu zu definieren, was klinisch wichtig ist. Dies meint neben der strukturierten Befundung zum Beispiel auch die Erstellung gemeinsamer Konsensusempfehlungen für die Herzbildgebung.

Sie haben seit 2020 mit Herrn Dr. Malte Sieren ein kooptiertes Vorstandsmitglied aus den Reihen des Forums Junge Radiologie hinzugewonnen. Ist der radiologische Nachwuchs damit endgültig in der AG angekommen?

Und ob! Dr. Malte Sieren ist ein gutes Beispiel, aber auch Dr. Sebastian Reinartz als kooptiertes Mitglied möchte ich erwähnen, der sich sehr für Fortbildung und RemotePrüfungen mit Fallsammlungen engagiert. Dass auch noch erfahrenere Akteure dabei sind, ist meines Erachtens aber auch wichtig, wie zum Beispiel Professor Mathias Gutberlet als Organisator der Deutschen Kardiodiagnostiktage.

Haben Sie außerdem noch Pläne und Ideen für Ihre Amtszeit, die Sie uns jetzt schon verraten können?

Oberstes Ziel ist es, die Expertise unserer Community in der Herzbildgebung weiter zu fördern. Nur wenn wir richtig gut sind, werden wir unsere Position behaupten können. Außerdem müssen wir im ständigen Austausch bleiben mit allen Interessensgruppen, in der DRG und außerhalb. Für die erfolgreiche Zusammenarbeit, die viel Spaß macht, danke ich an dieser Stelle allen Beteiligten Vorstandsmitgliedern und kooptierten Kolleginnen sowie Kollegen, den Mitarbeiterinnen und Mitarbeitern der Geschäftsstelle und allen beteiligten Mitgliedern der AG.

\section{Vielen Dank für das Interview!}

www.ag-herz.drg.de 